\title{
Development of Automotive Media System Evaluated on Compliance Test
}

\author{
Sang Yub Lee*, Duk Geun Park*, Jae Kyu Lee* \\ *Software Device Research Centre, Korea Electronics Technology Institute, Republic of Korea \\ syublee@keti.kr, parkdk@keti.kr,jae4850@keti.kr
}

\begin{abstract}
- among the variety of vehicle technology trend issues, the biggest issue is focus on automotive network system. Especially, optical network system is preferred. The aim in optical network system including vehicular environment information is that realization of media streaming service beyond the current media system. This paper is introduced the implementation of media streaming service including optical network as called MOST (Media Oriented System Transport) and realization of car audio system adapted to vehicular environment information collected from OBD (On-Board Diagnosis) via CAN (Controller Area Network). Especially on the reliability of vehicular network system, it is executed the MOST compliance test and showed the network test result.
\end{abstract}

Keywords - In-vehicle network, MOST, OBD, automotive media system

\section{INTRODUCTION}

With the passage of time, vehicle technology in modern times has been developed rapidly. Recent of today's automobile research area has been gradually changed from mechanics to electronics to the way of offering entertainment service to customs that can serve the connection to smart device easily and conveniently.

In particular, most of people want to be experienced in high quality audio streaming service while they drive. According to customer's demands, MOST system is developed to provide an efficient and cost effective fabric to transmit audio data between any devices attached to the harsh environment of automobile. Compared to conventional car audio system which has been used in many of audio electrical line, bundle of lines per an audio channel, dissimilarly being with the one optical line in order to transit the audio data source, the advanced streaming service adopted optical network system makes light harness system. As the simply network construction, it can be obtained the high fuel efficiency from the usage of plastic optical fiber and for optical signal characteristics, it makes be free on electro-magnetic problems. Considered on driving environment, car audio system as the streaming service gathering automobile information via CAN enables to tune the volume automatically for their vehicular conditions. To develop the self-contained audio system, the complex information having vehicular status is needed to be accessed more comfortable.
In Section 2, MOST network system and MOST data processing are introduced as in-vehicle networks system. The vehicle information processing module which classified into vehicular status information to be collected from CAN network is de-scribed, and demonstration of streaming service are shown. This paper is concluded in Section 3.

\section{NETWORK SYSTEM}

\section{A. MOST System}

MOST is the de-factor standard for efficient and cost effective networking of automotive multimedia and infotainment system [1]-[2]. The current MOST standards released MOST150, 150 means that $150 \mathrm{Mbps}$ network bandwidth with quality of services is available in Figure 1. In described in Figure.2, proposed network system is consist of MOST devices with CAN bus line. In conception of the network topology, MOST devices are divided into master and slave mode. Master mode device with CAN bus can be realized the optimum sound effect depending on vehicular environment information gathered by OBD interface in car.

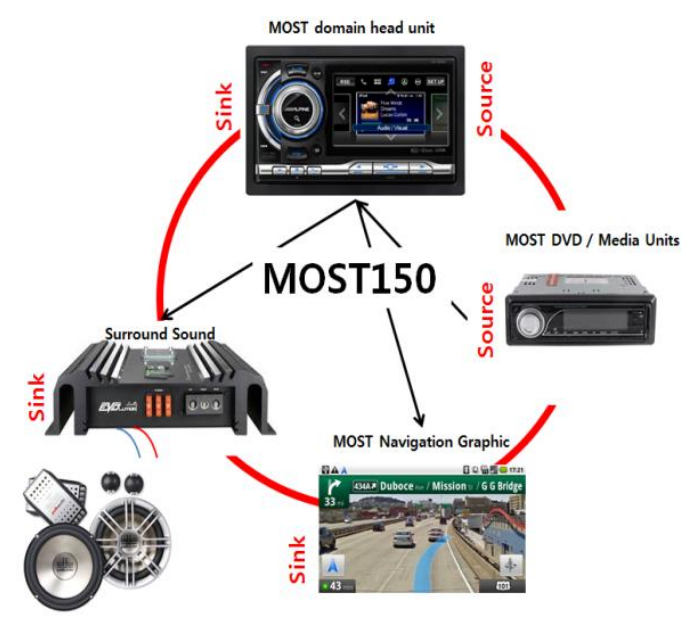

Figure 1.MOST Network System

1) MOST Network Services for External Host Controller: Network service is based on the network interface controller and provides a programming interface for the application which basically consists of the function blocks. In this paper, 
it comprise designed platform for transferring streaming data in synchronous channel. It contains mechanisms and routines for operating and managing the network and it ensures dynamic behaviour of the network. The network service is implemented on the external host controller as shown in Figure. 2.

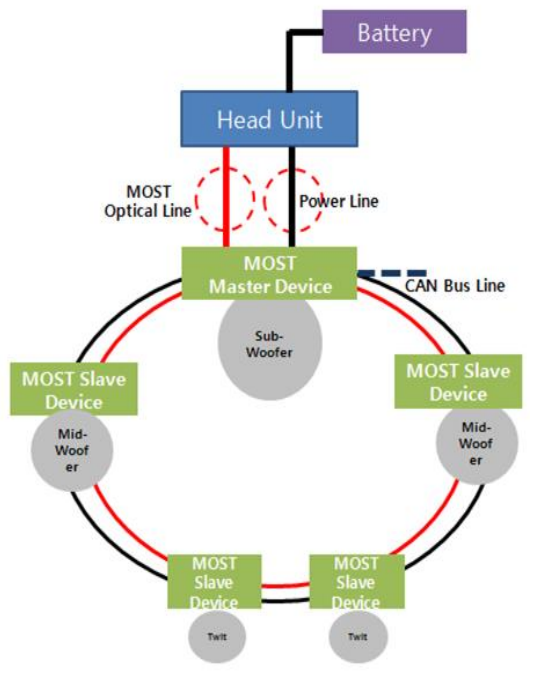

Figure 2. MOST Network System with CAN bus line

2) MOST Streaming Data Processing; for the data processing, it is explained in streaming data part and network service one. Being presented the streaming data part; the bandwidth of the streaming data channel can be calculated using the following formula

\section{Bandwidth $=93 \times 8$ bit $\times 48 \mathrm{kHz}$}

Synchronous data is used for the real-time transmission of audio data. Before data transmission, the data transmitted for the synchronous connection must be established by the connection master in network system. For this method, one socket has to be created and connected at the interfaces to the frame and to the local resource. Thus, up to 93 stereo connections can be established simultaneously on a MOST 150 frame. The content of the frame remains unmodified until the frame arrives back at the sending node. The quasi-static establishment of connections on a channel is denominated as TDM (Time Division Multiplexing). The data are transmitted cyclically in a specified time pattern at the same frame position. There is no repetition in the case of communication errors. A valid value is then available the next cycle.

On the network service side, MOST devices have the unique interface which transfers the data between the physical layer network and processor as an external host controller [4]. Working in conjunction with the clock manager, the network port recovers the network clock for time synchronization. And then, receiving data is decoded and delivered to the microprocessor. Transferred data is routed into appropriate memory destination on and off in platform.

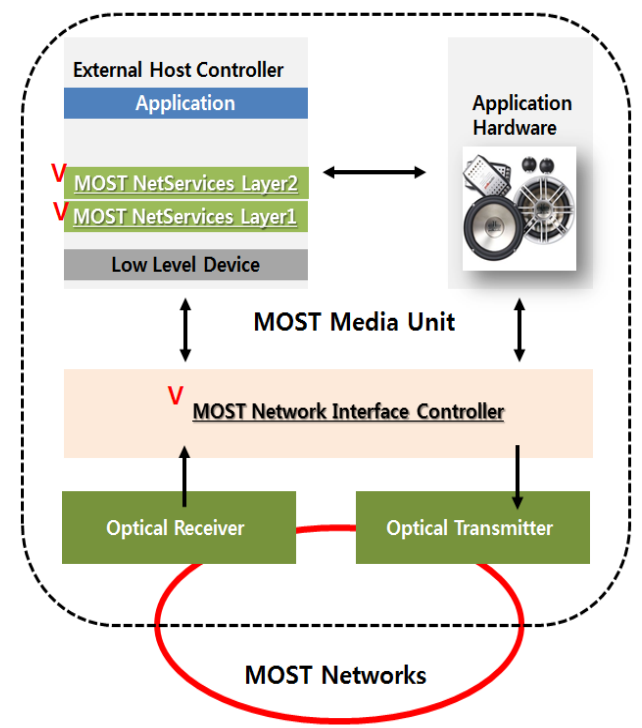

Figure 3. MOST Media Device Unit

\section{B. MOST Compliance Test}

The integration of network system and modules is particularly challenging due to their complexity. An effective measure is to introduce a certification test process so that the devices and modules are tested before they are integrated into the system. As described in Figure. 4, MOST compliance test process is divided into three courses: device test on physical layer, higher communication levels and application layer.

With regard to the physical layer compliance, the measurement point is to check the signal characteristics for constancy. For the normal operation, power, error, ring break diagnosis and network management, higher communication level compliance tests are performed. Distinctive features and the dynamic behaviours are tested in the application layer compliance test.

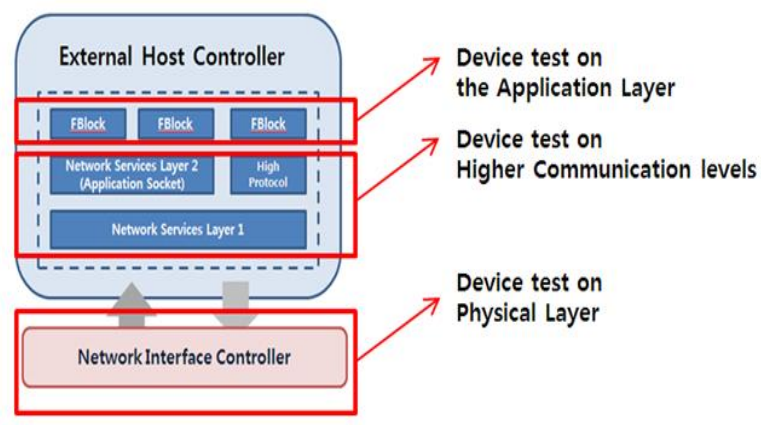

Figure 4. MOST Compliance Test on the Media Device Unit

\section{Vehicular Information}

Vehicular sensing module as depicted in Figure. 5 is coded into vehicular status information and status transit method. For applications, expressed black box on right side in Figure 5, vehicle processing module is displayed engine rpm, vehicle speed, gear information, battery status and engine load to panel of platform. 


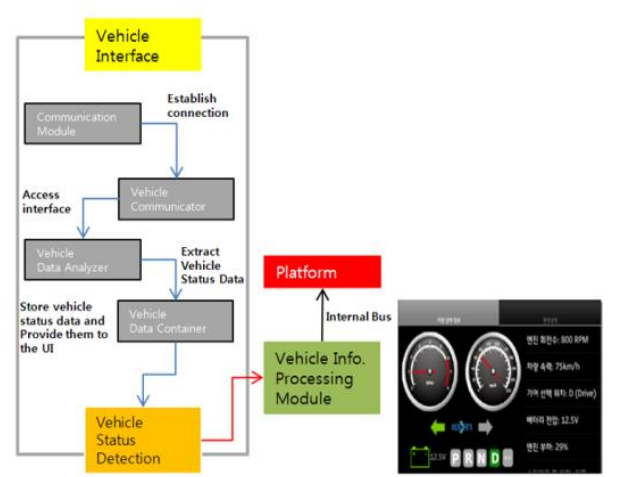

Figure 5. CAN Data Processing Module

\section{III.IMPLEMENTATION OF AUTOMOTIVE MEDIA PLATFORM}

\section{A. Platform Architecture}

The external host controller communicates with network interface controller via I2C bus and connected with OBD via CAN bus [5]-[6]. As shown below, the streaming port included in network interface controller can be used for stereo audio exchange between the network and physical audio port. The external host controller may support for managing the streaming audio exchange remotely.

As described in Figure. 6, in order to make the data connection path, Data transferred from the internal bus accesses into embedded memory space. External host controller is used for low graded microprocessor and internal local bus. It is designed as a platform shown in Figure. 7.

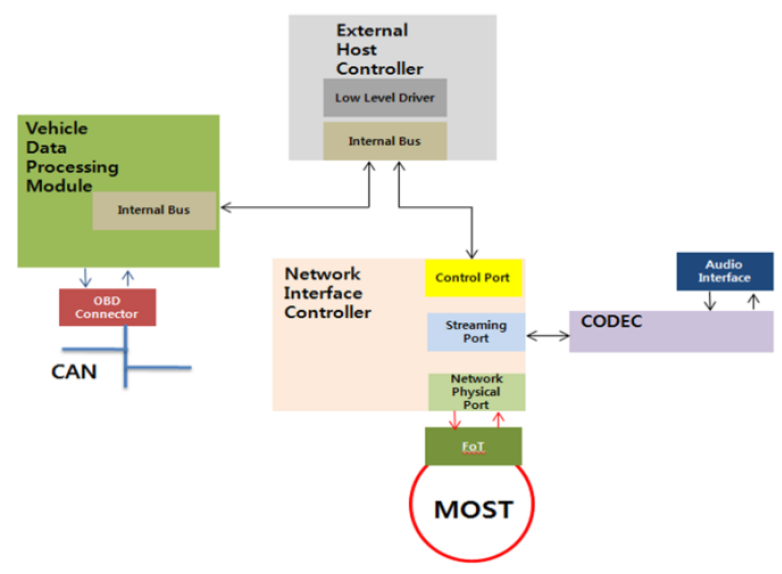

Figureure 6. The structure of media platform

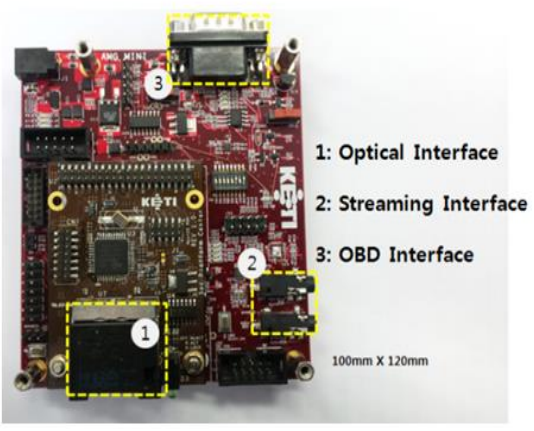

Figure 7. Media Service Platform

\section{B. System Demonstration}

As shown in Figure. 8, system demonstration is built with designed platform, audio platform connected with audio speaker and amplifier, CAN frame generator which makes the virtual environment via OBD interface applied to car. With running this set, it realizes that driver and passenger experience the car audio sound depending on vehicular status automatically without voluntary control.

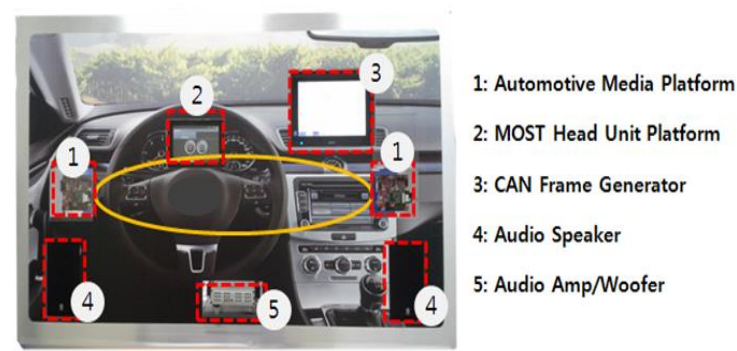

Figure 8. System Demonstration

\section{Compliance Test Result}

Figure 9 shows the test result for physical layer compliance for MOST150. Based on an analysis of the higher communication level, the compliance test cases were determined as network behaviour, system configuration and notification method in Figure. 10.

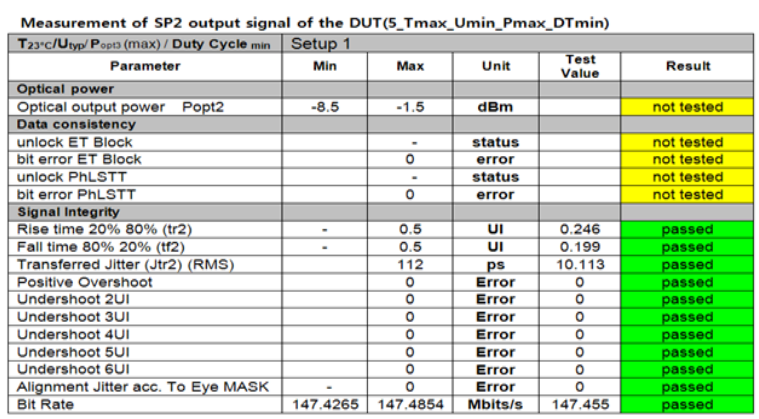

Figure 9. The result of physical layer compliance test 


$\begin{gathered}\text { expected } \\ \text { result } \\ \text { DUT responds correctly to all queries }\end{gathered}$
DUT responds correctly to all queries
DUT ok
Thesult
The DUT has passed the test

Figure 10. The summary of higher communication level compliance test on MOST network system

\section{IV.CONCLUSIONS}

This paper is introduced the development of the automotive media platform based on optical network system. Particularly, optical network system, MOST, to be optimized sound level depending on vehicle environment is satisfied with reducing the weight and ensuring the reliability for free of electromagnetic problems. With vehicle status information, designed network platform realized and demonstrated that selfcontained MOST speaker and woofer can be controlled and tuned their volume level automatically to be served to passengers more conveniently. For being ensured reliability of designed platform, it is shown compliance test of physical and higher communication level

\section{ACKNOWLEDGMENT}

This work was supported by the IT R\&D program of MKE/KEIT [R0001939, Development of the 150Mbps class optical device applied automotive streaming service system]

\section{REFERENCES}

[1] Andreas Grzemba, "MOST Book from MOST25 to MOST150," MOST Cooperation, FRANZIS 2012.

[2] Otto Strobel, Rindha Rejeb and Jan Lubkoo, "Communication in Automotive System Princi-ples, Limits and New Trends for Vehicles, Airplanes and Vesseles," IEEE ICTON, pp. 1-6, 2007.

[3] M. Wegmuller, J. P. von der Weid, P. Oberson, and N. Gisin, "High resolution fiber distrib-uted measurements with coherent OFDR," Proc. of ECOC, pp. 109, Jan. 2000.

[4] Sang Yub Lee, "A method of vehicular data collection and processing using wireless communications interface," KICS conference, 2010.

[5] S. Godavarty, S. Broyles, M. Parten, "Interfacing to the on-board diagnostic system," Proc. of IEEE 52nd-VTC, pp. 24, 2000.

[6] Sang Yub Lee, Sang Hyun Park, Hyo Sub Choi and Chul Dong Lee "MOST Network Sys-tem Supporting Full-Duplexing Communication," IEEE ICACT, pp.1272-1275, 2012. 